\title{
Additive Schwarz method for scattering problems using the PML method at interfaces
}

\author{
Achim Schädle ${ }^{1}$ and Lin Zschiedrich ${ }^{2}$ \\ 1 Zuse Institute, Takustr. 7, 14195 Berlin, Germany schaedle@zib.de \\ 2 Zuse Institute, Takustr. 7, 14195 Berlin, Germany zschiedrich@zib.de
}

\section{Introduction}

Exterior Helmholtz problems are a basic model for wave propagation in the frequency domain on unbounded domains. As a rule of thumb, 10-20 grid points per wavelength are required. Hence if modeling structures that are a multiple of wavelengths in size, a discretization with finite elements results in large sparse indefinite and unsymmetric problems. There are no well established solvers, or preconditioners for these linear systems as there are for positive definite elliptic problems.

As a first basic step towards a solver for the class of linear systems described above we consider a non-overlapping Schwarz algorithm with only two sub-domains, where the coupling among sub-domains is done using the perfectly matched layer method.

We do not present a new idea here, and it is beyond the scope of the paper to do justice to previous work in this field. However we comment on a few references, that have been of inspirational value to us.

In [11] Toselli tried to use the Schwarz algorithm with perfectly matched layers (PML) at the interfaces, as a preconditioner. However the coupling of the incoming waves there, was done in the wrong way, we comment on this in the concluding remark in Section 4. One may view the ansatz by Despres, see [1] and the references therein, and Shaidurov and Ogorodnikov [10], as a first order absorbing boundary condition. The use of Robin boundary conditions there is also motivated by the idea of equating energy fluxes over boundaries. Colino, Joly and Ghanemi [3] analyzed the ansatz by Despres and could prove convergence. Gander, Nataf and Magoulés [5] follow a slightly different ansatz. They use local low order boundary conditions, that optimize transmission, based on the analysis of Fourier coefficients.

The PML method is in special cases one of the best approximations to the Dirichlet to Neumann (DtN) operator. With the DtN operator at hand the Schwarz algorithm would converge in a finite number of iteration steps. 


\section{Problem description}

We consider time-harmonic electro-magnetic scattering problems in two space dimensions. Assuming that the electric field is polarized in the $x, y$-plane and that the obstacle is homogeneous in the $z$ direction, the time-harmonic vectorial Maxwell's equations in 3D are reduced to equations in 2D. For the $z$ component of the magnetic field we obtain the Helmholtz equation (1)

$$
\nabla \cdot \epsilon^{-1} \nabla u+\omega^{2} \mu u=0 \text { in } \tilde{\Omega} ; \quad b\left(u, \partial_{\nu} u\right)=0 \text { on } \Gamma
$$

Here $\omega$ is the frequency and $\mu$ and $\epsilon$ are the $x, y$-dependent relative permeability and conductivity respectively. $\tilde{\Omega}$ is typically the complement of a bounded set in $\mathbb{R}^{2}$, with boundary $\Gamma$, where the boundary condition $b$ is given. The boundary condition $b$, if there is an interior boundary at all, is typically of the form $b\left(u, \partial_{\nu} u\right)=u, b\left(u, \partial_{\nu} u\right)=\partial_{\nu} u$ or $b\left(u, \partial_{\nu} u\right)=\partial_{\nu} u+c u$. The Helmholtz equation has to be completed by the Sommerfeld radiation boundary condition for the scattered field.

For simplicity we assume that $\epsilon=1$, and set $k^{2}=\omega^{2} \mu$. The total field $u$ can be written as the sum of the known incoming and the scattered field $u=$ $u_{i n}+u_{s c}$. The scattered field is a solution of (1) and satisfies the Sommerfeld radiation boundary conditions for $|(x, y)| \rightarrow \infty$ given by:

$$
\lim _{|(x, y)| \rightarrow \infty} \partial_{\nu} u_{s c}=i k u_{s c},
$$

where the limit is understood uniformly for all directions.

\section{Coupling of incoming waves - DtN operator}

The computation will be restricted to a bounded computational domain $\Omega$. It is assumed that outside the computational domain $\epsilon$ and $\mu$ are constant along straight lines. In this case we can evaluate the Dirichlet to Neumann (DtN) operator using the perfectly matched layer method (PML) developed in [13].

Next we reformulate the Problem (1) on the computational domain. This clearly shows how to couple incoming fields to the computational domain.

Setting $u=v \oplus w$ according to the decomposition $\tilde{\Omega}=\Omega \cup \Omega_{\text {ext }}$ we obtain the coupled system

$$
\begin{gathered}
\Delta v+k^{2} v=0 \text { in } \Omega ; \quad b\left(v, \partial_{\nu} v\right)=0 \text { on } \Gamma \cap \Omega \\
\partial_{\nu} v=\partial_{\nu} u_{i n}+\partial_{\nu} w_{s c} \text { on } \Gamma_{\text {int }} \\
\Delta w_{s c}+k^{2} w_{s c}=0 \text { in } \Omega_{e x t} ; \\
w_{s c}=v-u_{i n} \text { on } \Gamma_{i n t} ; b\left(w_{s c}+u_{i n}, \partial_{\nu}\left(w_{s c}+u_{i n}\right)\right)=0 \text { on } \Gamma \cap \Omega_{e x t} \\
\lim _{|x| \rightarrow \infty} \partial_{\nu} w_{s c}-i k w_{s c}=0
\end{gathered}
$$


where the coupling is via the Dirichlet and Neumann data on the interface boundary $\Gamma_{\text {int }}$, connecting $\Omega$ and $\Omega_{\text {ext }}$. From this we obtain the DtN operator, which is the operator that solves the exterior problem with given Dirichlet data $\Gamma_{\text {int }}$ and returns the Neumann data. With the DtN-operator at hand one get

$$
\begin{aligned}
\Delta v+k^{2} v & =0 \text { in } \Omega ; \quad b\left(v, \partial_{\nu} v\right)=0 \text { on } \Gamma \cap \Omega \\
\partial_{\nu} v-\partial_{\nu} u_{i n} & =\operatorname{DtN}\left(v-u_{i n}\right) \text { on } \Gamma_{\text {int }} .
\end{aligned}
$$

In general the DtN operator is hard to get, but can be approximated using the PML, described briefly in Section 4. For more information on approximating the DtN operator, see the textbook [6], and the more recent review articles [12, $7]$.

\section{Sketch of the perfectly matched layer method}

We do not follow, the classical introduction of the perfectly matched layer method (PML) that is motivated by adding a layer of artifical absorbing material.

Our derivation of the PML method, described in detail in [8] is based on an analytic continuation, as in $[9,2]$ Details of the implementation in 2D can by found in [13]. The basic idea is an analytic continuation of the solution in the exterior along a distance variable. We will only sketch the ideas here for the one-dimensional case.

Consider the Helmholtz equation in 1D on a semi-infinite interval for the scattered field.

$$
\begin{aligned}
\partial_{x x} u+k^{2} u & =0 \quad x \in[-1, \infty) \\
u(-1) & =1 ; \quad \partial_{\nu} u=i k u \text { for } x \rightarrow \infty
\end{aligned}
$$

Our computational domain is the interval $[-1,0]$. The solution in the exterior is analytic in $x$. Defining $\gamma(x):=(1+i \sigma) x$ and $\tilde{u}_{\text {PML }}(x):=u(\gamma(x))$, we have $\tilde{u}_{\mathrm{PML}}(0)=u(0)$ and $\partial_{\nu} u(0)=\partial_{\nu} \tilde{u}_{\mathrm{PML}}(0) /(1+i \sigma) \cdot u_{\mathrm{PML}}$ obeys

$$
\begin{aligned}
\partial_{x x} \tilde{u}_{\mathrm{PML}}+k^{2}(1+i \sigma)^{2} \tilde{u}_{\mathrm{PML}} & =0 \quad x \in[0, \infty) \\
\tilde{u}_{\mathrm{PML}}(0)=u(0) ; \quad \partial_{x} \tilde{u}_{\mathrm{PML}}(x) & =i k \tilde{u}_{\mathrm{PML}}(x)(1+i \sigma)
\end{aligned}
$$

Fundamental solutions are $\exp (i k(1+i \sigma) x)$ and $\exp (-i k(1+i \sigma) x)$. The first one is called outgoing as it obeys the boundary condition, the second is called incoming as it does not obey the boundary condition. The first one is decaying exponentially, whereas the second is growing exponentially, therefore it can be justified to replace $\tilde{u}_{\mathrm{PML}}$ by $u_{\mathrm{PML}}$ given by Equation (9), and replace the infinite coupled system by the coupled system

$$
\begin{gathered}
\partial_{x x} v+k^{2} v=0 \quad x \in[-1,0] \\
v(-1)=1 ; \quad \partial_{\nu} v(0)=\partial_{\nu} u_{\mathrm{PML}}(0) /(1+i \sigma)
\end{gathered}
$$




$$
\begin{array}{r}
\partial_{x x} u_{\mathrm{PML}}+k^{2}(1+i \sigma)^{2} u_{\mathrm{PML}}=0 \quad x \in[0, \rho] \\
u_{\mathrm{PML}}(0)=v(0) ; \quad \partial_{x} u_{\mathrm{PML}}(\rho)=0
\end{array}
$$

Here $\rho$ is the thickness of the PML. The error introduced by cutting the PML is analyzed for example in $[9,8]$, where it is shown that the PML system is well-posed and the error decays exponentially with $\rho$.

Remark: Toselli [11] coupled the incoming field at the external boundary of the PML, this way the incoming field is damped in the PML and this might explain, why he concluded that it is best to use a very thin layer.

\section{Two-domain decomposition}

We now turn back to the two dimensional case. The idea for the Schwarz algorithm is to calculate the solution on every sub-domain separately with transparent boundary conditions and add the scattered field of one domain to the incoming field for the neighboring domains, i.e. we use some sort of Pseudo-DtN operator, where we assume that the exterior to each sub-domain has a simple structure. If we are able to evaluate the DtN operator the Schwarz algorithm would converge in a finite number of steps.

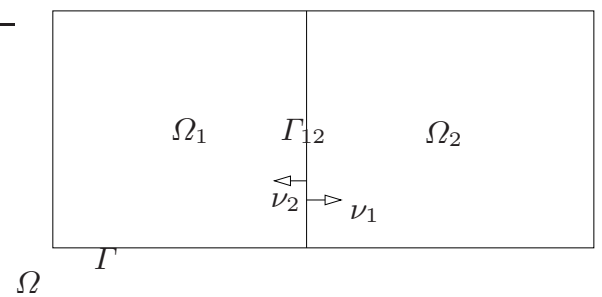

Fig. 1. Decomposition of $\Omega$ into two non-overlapping sub-domains $\Omega_{1}$ and $\Omega_{2}$

For the simple two sub-domain case the additive Schwarz algorithm is given in (10). There $u_{j}^{n}$ denotes the $n$th iterate on sub-domain $\Omega_{j}$, and $\Gamma_{i j}$ the boundary between $\Omega_{i}$ and $\Omega_{j}$.

$$
\begin{aligned}
& \Delta u_{j}^{n+1}+k^{2} u_{j}^{n+1}=0 \text { in } \Omega_{j} \\
& \partial_{\nu} u_{j}^{n+1}=\operatorname{DtN}\left(u_{j}^{n+1}-u^{i n}\right)+\partial_{\nu} u^{i n} \text { on } \bar{\Omega}_{j} \cap \Gamma \\
& \partial_{\nu} u_{j}^{n+1}=\operatorname{DtN}\left(u_{j}^{n+1}-u_{i}^{n}\right)+\partial_{\nu} u_{i}^{n} \text { on } \Gamma_{i j}
\end{aligned}
$$

for $(i, j)=(1,2),(2,1), n=0,1, \ldots$

Denoting by $\nu_{j}$ the normal with respect to $\Omega_{j}$ we have $\partial_{\nu_{j}} u_{i}^{n}=-\partial_{\nu_{i}} u_{i}^{n}$.

Restrictions:

sub-domains ordered linearly, strip with homogenous Neumann, Dirichlet, or periodic boundary conditions cut in slices This way we avoid crosspoints, 
which pose a problem. The incoming field is given on two neighboring domains with a common boundary, hence the incoming field may have a jump across this boundary and at the crosspoint, and is hence not a solution of the Helmholtz equation. This is also a problem from the computational point of view, as the Dirichlet data inserted in the DtN operator is assumed to be continuous. One idea to circumvent this difficulty is to add artificial outgoing waves, that compensate for the jump. Another one is to use a representation formula based on the Pole condition for the scattered field and evaluate it on the interface boundaries, but this is out of the scope of the present paper.

We assume that the boundary condition is a homogenous Neumann condition, i.e. $b\left(u, \partial_{\nu} u\right)=\partial_{\nu} u$, and set

$$
a_{\Omega}(u, \varphi)=-\int_{\Omega} \nabla u \nabla \varphi+k^{2} u \varphi d x
$$

With this in the variational setting the solution $u$ is the function $u \in H^{1}(\Omega)$ such that

$$
a_{\Omega}(u, \varphi)+\int_{\Gamma_{\text {int }}} \partial_{\nu} u \varphi d \sigma(x)=0 \quad \forall \varphi \in H^{1}(\Omega)
$$

Inserting the boundary condition, we obtain

$$
a_{\Omega}(u, \varphi)+\int_{\Gamma_{\text {int }}} \operatorname{DtN}\left(u-u^{i n}\right) \varphi+\partial_{\nu} u^{i n} \varphi d \sigma(x)=0 \quad \forall \varphi \in H^{1}(\Omega)
$$

The Schwarz algorithm in variational form is given in (12) below. To avoid the evaluation of the Neumann data on the interface boundary we use a postprocessing step (13), so that the Neumann data is only given in weak form.

$$
\begin{gathered}
a_{j}\left(u_{j}^{n+1}, \varphi\right)+\underbrace{\int_{\Gamma_{i j}} \operatorname{DtN}\left(u_{j}^{n+1}-u_{i}^{n}\right) \varphi d \sigma(x)+\int_{\Gamma_{i j}} \partial_{\nu_{j}} u_{j}^{n} \varphi d \sigma(x)}_{\int_{\Gamma_{i j}} \partial_{\nu_{1}} u_{1}^{n+1} \varphi d \sigma(x)} \\
+\underbrace{\int_{\Gamma \cap \bar{\Omega}_{j}} \operatorname{DtN}\left(u_{j}^{n+1}-u^{i n}\right) \varphi+\partial_{\nu_{1}} u^{i n} \varphi d \sigma(x)}_{\int_{\Gamma \cap \bar{\Omega}_{j}} \partial_{\nu_{j}} u_{j}^{n+1} \varphi d \sigma(x)}=0 \quad \forall \varphi \in H^{1}\left(\Omega_{1}\right) \\
\int_{\Gamma_{i j}}^{\partial_{\nu_{j}} u_{j}^{n+1} \varphi d \sigma(x)=-a_{j}\left(u_{j}^{n+1}, \varphi\right)} \\
-\int_{\Gamma \cap \bar{\Omega}_{j}} \operatorname{DtN}\left(u_{j}^{n+1}-u^{i n}\right) \varphi+\partial_{\nu_{1}} u^{i n} \varphi d \sigma(x)
\end{gathered}
$$

\section{Numerical experiments}

We consider a very simple example. The computational domain is a $[-1,1] \times$ $[0.5,0.5]$ rectangle, with periodic and transparent boundary conditions. To be 
precise, in Fig. 1 we take periodic boundary conditions at the top and bottom of $\Omega$ and transparent boundary conditions to the left and the right. The incoming field is a plane wave traveling from left to right. The computational domain is split in two squares along the $y$-axis. The $x, y$ dependent $k$ is a step function, $k$ equals $k_{0}$ everywhere, except in two smaller squares of size $[0,0.5] \times[0,0.5]$ located in the center of the two sub-domain, where it is $k_{0} / 5$.

The calculation was done using the package JCMfeetools developed at the ZIB, with second order finite elements. The linear systems are solved using the sparse solver UMFPACK.

The thickness of the PML $\rho$ is set to three wavelengths, the damping factor $\sigma=1$ and along the distance variable, we have chosen 12 grid-points on the coarse grid. The coarse grid including the PML has about 1100 unknowns on each sub-domain. We plot the $l_{2}$ error versus number of Schwarz iteration steps for different $\omega$ for upto four uniform refinements of the initial grid. To this end the error is calculated with respect to a reference solution calculated on the whole domain with the same mesh on each subdomain. This is done for two settings. First for the algorithm described above, with the representation of the Neumann data in weak form and second evaluating the normal derivatives, via the gradient of the ansatz function in the neighboring domain.

In case we use the weak representation of the Neumann data, we obtain a convergent algorithm. The convergence rate depends strongly on the wavelength but only weakly on the discretization as can be seen in Fig 2 .
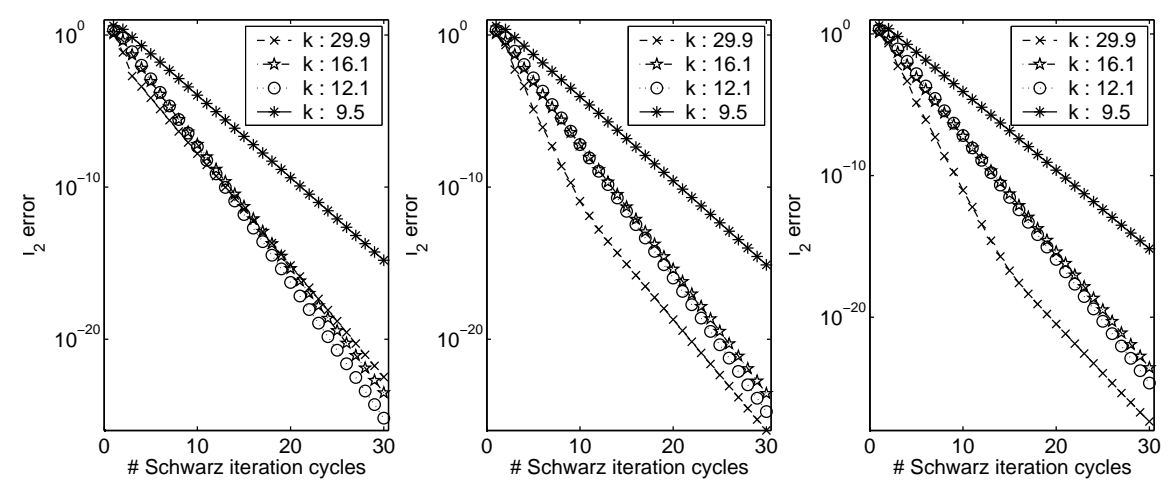

Fig. 2. Error of the Schwarz algorithm, for different wavenumbers $k$ and different refinement levels using weak representation of the Neumann data The plot at the left was calculated using 1168 unknowns, the one in the middle with 4000 unknowns and the one to the right with 13120 unknowns.

In case we evaluate the Neumann data via the gradient of the ansatz function the error of the domain decomposition method saturates as shown in the left and middle graphics in Fig 3. 

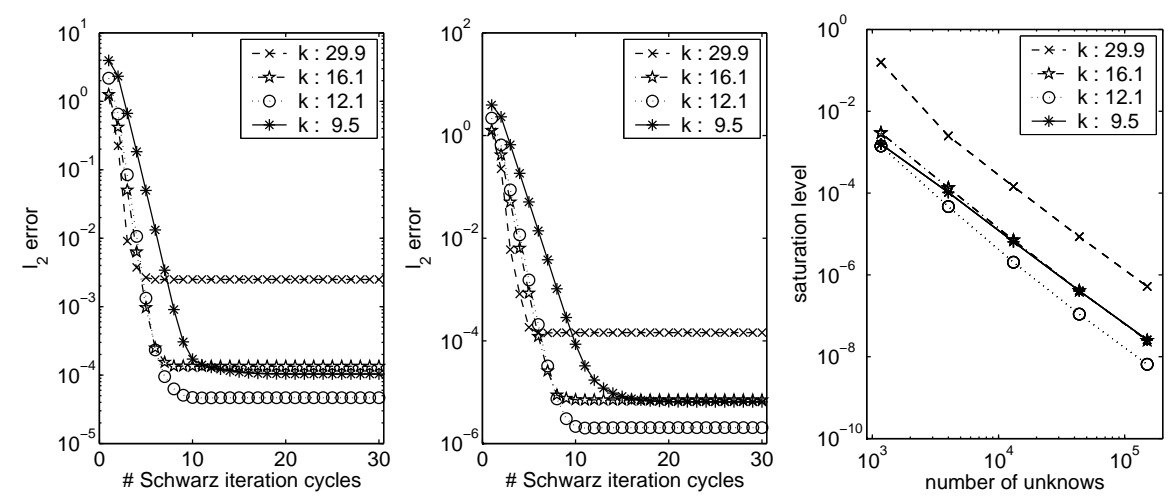

Fig. 3. (Left and middle): Error of the Schwarz algorithm, for different wavenumbers $k$ and different refinement levels. The plot at the left was calculated using 4000 unknowns the one in the middle with 13120 unknowns on each sub-domain. (Right): Decay of the level at which the error saturates, versus the number on unknowns.

Surprisingly, the level at which the error saturates, plotted in the most right graphic in Fig 3 versus the number of unknowns, decays faster than might be expected, from the error estimate for the Neumann data. Remember that we use second order finite elements here.

\section{Acknowledgment}

We thank Ralf Kornhuber, Susanne Ertel and the members of the computational nano optics group for fruitful discussions and special thanks to Martin Gander and Ralf Hiptmair. The first author was supported by the DFG Research Center Matheon "Mathematics for key technologies", Berlin. The second author was supported by the German Federal Ministry of Education and Research BMBF project under contract no 13N8252 (HiPhocs).

\section{References}

1. J.-D. Benamou and B. Després. A domain decomposition method for the helmholtz equation and related optimal control problems. J. Comput. Phys., 136(1):68-82, 1997.

2. F. Collino and P. Monk. The perfectly matched layer in curvilinear coordinates. SIAM J. Sci. Comput., 1998.

3. Francis Collino, Souad Ghanemi, and Patrick Joly. Domain decomposition method for harmonic wave propagation: A general presentation. Comput. Methods Appl. Mech. Eng., 184(2-4):171-211, 2000.

4. Bjorn Engquist and Hong-Kai Zhao. Absorbing boundary conditions for domain decomposition. Appl. Numer. Math., 27(4):341-365, 1998. 
5. Martin J. Gander, Frdric Magouls, and Frdric Nataf. Optimized Schwarz methods without overlap for the Helmholtz equation. SIAM J. Sci. Comput., 24(1):38-60, 2002.

6. D. Givoli. Non-reflecting boundary conditions. J. Comput. Phys., 94(1):1-29, 1991.

7. T. Hagstrom. Radiation boundary conditions for numerical simulation of waves. Acta Numerica, 8:47-106, 1999.

8. Thorsten Hohage, Frank Schmidt, and Lin Zschiedrich. Solving Time-Harmonic Scattering Problems Based on the Pole ConditionII: Convergence of the PML Method. SIAM J. Math. Anal., 35(3):547-560, 2003.

9. M. Lassas and E. Somersalo. On the existence and convergence of the solution of PML equations. Computing, 60(3):229-241, 1998.

10. V. V. Shaidurov and E. I. Ogorodnikov. Some numerical method of solving helmholtz wave equation. In G. Cohen, L. Halpern, and P. Joly, editors, Mathematical and numerical aspecst of wave propagation phenomena, pages 73-79. SIAM, 1991.

11. A. Toselli. Some results on overlapping schwarz methods for the helmholtz equation employing perfectly matched layers. Technical Report Technical Report 765, Courant Institute, New York University, June 1998.

12. S. Tsynkov. Numerical solution of problems on unbounded domains. A review. Applied Numerical Mathematic, 27:465-532, 1998.

13. Lin Zschiedrich, Roland Klose, Achim Schdle, and Frank Schmidt. A new finite element realization of the Perfectly Matched Layer Method for Helmholtz scattering problems on polygonal domains in 2D. Technical Report 03-44, ZIB, 2003. 